\title{
OPTIMAL RESOURCE ALLOCATION IN OFDMA DOWNLINK SYSTEMS WITH IMPERFECT CSI
}

\author{
Rohit Aggarwal ${ }^{*}$, Mohamad Assaad $^{+}$, C. Emre Koksal*, and Philip Schniter* \\ *Dept. of ECE, The Ohio State University, Columbus, OH 43210. \\ ${ }^{+}$Supélec, Gif sur Yvette, France. \\ \{aggarwar,koksal, schniter\}@ece.osu.edu, \\ mohamad.assaadesupelec.fr
}

\begin{abstract}
In this paper, we address the problem of joint scheduling and resource allocation in the downlink of an orthogonal frequency division multiple access (OFDMA)-based wireless network when the per-user SNR is known only in distribution. In particular, we consider sum-utility maximization over user schedules, powers, and code rates, subject to an instantaneous sum-power constraint. We consider both a "continuous" scenario where, during a time-slot, each OFDMA subchannel can be time-shared among multiple users and/or code rates, and a "discrete" scenario where no time-sharing is allowed. For the non-convex optimization problem arising in the continuous case, we propose an efficient exact solution. For the mixedinteger optimization problem arising in the discrete case, we propose a polynomial-complexity approximate solution and derive a bound on its optimality gap. We also provide a numerical study of goodput maximization for the SNR distribution that results from the use of pilot-aided MMSE channel estimation.
\end{abstract}

\section{INTRODUCTION}

In the downlink of a wireless orthogonal frequency division multiple access (OFDMA) system, the base station (BS) delivers data to a pool of users whose channels vary in both time and frequency. Since bandwidth and power resources are limited, the BS must allocate them efficiently. At the same time, the BS may need to ensure quality-of-service (QoS) constraints, such as a minimum reliable rate for each user. Clearly, the optimal allocation of resources is a function of the instantaneous channel state of all users at all subchannels. However, it is difficult in practice to maintain perfect instantaneous channel state information (CSI) at the BS, and so resource allocation must be accomplished under imperfect CSI.

In this paper, we consider the problem of simultaneous userscheduling, power-allocation, and rate-optimization in an OFDMA downlink system when the BS knows the per-user SNR only in distribution. In particular, we consider the problem of maximizing expected sum-utility subject to a constraint on sum-power under two scenarios. In the first scenario, we allow multiple users and/or code rates to time-share each subchannel and time slot, resulting in a nonconvex optimization problem. We solve this problem using a dual optimization approach that yields an algorithm converging exponentially fast to the exact solution. In the second scenario, we allow at most one user-rate combination to be allocated on any subchannel, resulting in a mixed-integer optimization problem. We discuss the connection between the two scenarios and propose an approximate solution for the second problem using the solution obtained in the first. For some cases, we show that the proposed solution has zero optimality gap, while for the other cases, we bound the optimality gap. Finally, we describe numerical results investigating sum-goodput maximization in the OFDMA downlink where partialCSI is obtained through pilot-aided MMSE channel estimation. We note that this paper is an abbreviated version of a longer paper [1] that contains proofs and additional results.

We now discuss related work. The problem of OFDMA downlink scheduling and resource allocation under perfect CSI has been widely addressed in a number of publications (e.g., [2-5]). The effect of imperfect CSI is studied for single-user OFDM in [6-8]. In [6], channel prediction was used to mitigate the effect of outdated CSI on the performance of adaptive OFDM systems. The effect of OFDM channel estimation error, as well as that of outdated CSI, were studied for the variable bit-rate case in [7]. In [8], an optimal power loading algorithm for rate maximization was derived based on average and outage capacity criteria, and it was concluded that the outage rate of the system may be greatly reduced due to CSI error. Multi-user OFDMA downlink performance under imperfect CSI has been studied in [9-11]. In [9], the authors considered the problem of ergodic weighted sum-rate maximization for user-scheduling and resource-allocation, and studied the impact of channel estimation error, where channel estimation error resulted from pilot-aided MMSE channel estimation. In [10], a cross-layer design was proposed to guarantee a fixed target-outage probability for slow-fading channels when pilots are used to obtain CSI and the users have heterogeneous delay requirements. In [11], the problem of total transmit power minimization, subject to strict constraints on conditional expected user capacities, was investigated. In contrast to these works, we focus on maximizing a more general concave goodput-based utility subject to a sum-power constraint when the imperfect CSI comes in the form of generic per-user SNR distributions.

\section{SYSTEM MODEL}

We consider a downlink OFDMA system with $N$ subchannels and $K$ active users $\left(N, K \in \mathbb{Z}^{+}\right)$. During every channel use, one codeword (from a generic signaling scheme) is transmitted using each OFDMA subcarrier. The OFDMA subchannels between the BS and each user are assumed to be non-interfering with gains that are timeinvariant over each codeword duration. Furthermore, the subchannels of each given user are assumed to be statistically independent of the channels of other users. Thus, the successful reception of a transmitted codeword depends on the corresponding subchannel's SNR $\gamma$, power $p$, and modulation and coding scheme (MCS) $m$. Here, we assume that MCS $m \in\{1, \ldots, M\}$ corresponds to a transmission rate of $r_{m}$ bits per codeword and a codeword error probability of the form $a_{m} e^{-b_{m} p \gamma}$, where $a_{m}$ and $b_{m}$ are known constants. Because we treat the subchannel SNR $\gamma$ as an exogenous parameter, the codeword error probability is a function of the received SNR $p \gamma$.

We denote the allocation decision variable by $I_{n, k, m}$, where 
$I_{n, k, m}=1$ means that subchannel $n$ is fully dedicated to user $k$ at MCS $m$, and $I_{n, k, m}=0$ means that subchannel $n$ is totally unavailable to user $k$ at MCS $m$. The subchannel resource constraint is then expressed as $\sum_{k, m} I_{n, k, m} \leq 1$ for all $n$. We denote $p_{n, k, m} \geq 0$ as the power that would be expended on subchannel $n$ if it was fully allocated to the user/rate combination $(k, m)$. Finally, we use $\gamma_{n, k}$ to denote the $n^{t h}$ subchannel's SNR for user $k$. We assume that the BS does not know $\gamma_{n, k}$ perfectly, but rather in distribution. Thus, our scheduling and resource allocation (SRA) problem can be written as

$$
\begin{aligned}
\mathrm{SRA} \triangleq & \max _{\substack{\left\{p_{n, k, m} \geq 0\right\} \\
\left\{I_{n, k, m} \geq 0\right\}}} \mathrm{E}\left\{\sum_{n=1}^{N} \sum_{k=1}^{K} \sum_{m=1}^{M} I_{n, k, m} \times\right. \\
& \left.U_{n, k, m}\left(\left(1-a_{m} e^{-b_{m} p_{n, k, m} \gamma_{n, k}}\right) r_{m}\right)\right\} \\
& \text { s.t. } \sum_{k, m} I_{n, k, m} \leq 1 \forall n \text { and } \\
& \sum_{n, k, m} I_{n, k, m} p_{n, k, m} \leq P_{\text {con }} .
\end{aligned}
$$

Here, the goodput $g=\left(1-a_{m} e^{-b_{m} p_{n, k, m} \gamma_{n, k}}\right) r_{m}$ represents the expected number of bits per codeword that can be transmitted without error, and the utility function $U_{n, k, m}(\cdot)$ is used to transform goodput into a quality-of-service (QoS) or fairness metric, e.g., maximin fairness or proportional fairness [12]. We allow $U_{n, k, m}(\cdot)$ to be any generic real-valued function that is twice differentiable, strictlyincreasing, and concave, with $\left|U_{n, k, m}(0)\right|<\infty$.

We consider two flavors of the SRA problem: a "continuous" one (termed CSRA) where any subchannel is allowed to be shared between multiple users-MCS combinations, i.e., $I_{n, k, m} \in[0,1]$ for all $n, k, m$, and a "discrete" one (termed DSRA) where subchannel sharing is not allowed, i.e., $I_{n, k, m} \in\{0,1\} \forall n, k, m$. Defining $\boldsymbol{I}$ as the $N \times K \times M$ matrix with $(n, k, m)^{\text {th }}$ element as $I_{n, k, m}$, the CSRA problem concerns (1) for $\boldsymbol{I} \in \mathcal{I}_{\text {CSRA, where }}$

$$
\mathcal{I}_{\text {CSRA }}:=\left\{\boldsymbol{I}: \boldsymbol{I} \in[0,1]^{N \times K \times M}, \quad \sum_{k, m} I_{n, k, m} \leq 1 \forall n\right\},
$$

whereas the DSRA problem concerns (1) for $I \in \mathcal{I}_{\text {DSRA, where }}$

$$
\mathcal{I}_{\text {DSRA }}:=\left\{\boldsymbol{I}: \boldsymbol{I} \in\{0,1\}^{N \times K \times M}, \sum_{k, m} I_{n, k, m} \leq 1 \forall n\right\} .
$$

In the next section, we discuss the CSRA and DSRA problems and highlight the relationship between them.

\section{OPTIMAL SCHEDULING AND RESOURCE ALLOCATION}

We first consider the CSRA problem, which is a non-convex optimization problem (due to the non-convex sum-power constraint). Fortunately, it can be converted into a convex optimization problem through the substitution $x_{n, k, m}=I_{n, k, m} p_{n, k, m}$, where $x_{n, k, m}$ is the "actual" power allocated to user $k$ at MCS $m$ on subchannel $n$ after scheduling. Taking this approach, we obtain

$$
\begin{aligned}
\mathrm{CSRA}= & \min _{\substack{\left\{x_{n, k, m} \geq 0\right\} \\
\boldsymbol{I} \in \mathcal{I}_{\mathrm{CSRA}}}} \sum_{n, k, m} I_{n, k, m} F_{n, k, m}\left(I_{n, k, m}, x_{n, k, m}\right) \\
& \text { s.t. } \sum_{n, k, m} x_{n, k, m} \leq P_{\text {con }},
\end{aligned}
$$

where $F_{n, k, m}(\cdot, \cdot)$ is given by

$$
\begin{aligned}
& F_{n, k, m}\left(y_{1}, y_{2}\right) \\
& = \begin{cases}-\mathrm{E}\left\{U_{n, k, m}\left(\left(1-a_{m} e^{-b_{m} \gamma_{n, k} y_{2} / y_{1}}\right) r_{m}\right)\right\} & \text { if } y_{1} \neq 0 \\
0 & \text { otherwise. }\end{cases}
\end{aligned}
$$

We denote the optimal $\boldsymbol{I}$ and $\boldsymbol{x}$ for (4) as $\boldsymbol{I}_{\text {CSRA }}^{*}$ and $\boldsymbol{x}_{\mathrm{CSRA}}^{*}$, respectively, and use $\boldsymbol{p}_{\mathrm{CSRA}}^{*}$ to denote the corresponding $\boldsymbol{p}$.

The convex problem (4) satisfies Slater's condition at $I_{n, k, m}=$ $\frac{1}{2 K M}$ and $x_{n, k, m}=\frac{P_{\text {con }}}{N} I_{n, k, m}, \forall n, k, m$. Hence, it can be solved using a dual optimization approach with zero duality gap [13]. Using $\mu$ as the dual variable, the Lagrangian of (4) is

$$
\begin{aligned}
L(\mu, \boldsymbol{I}, \boldsymbol{x})= & \sum_{n, k, m} I_{n, k, m} F_{n, k, m}\left(I_{n, k, m}, x_{n, k, m}\right)+ \\
& \left(\sum_{n, k, m} x_{n, k, m}-P_{\text {con }}\right) \mu,
\end{aligned}
$$

where $\boldsymbol{x}$ denotes the $N \times K \times M$ matrix $\left[x_{n, k, m}\right]$. The unconstrained dual problem then becomes

$$
\max _{\mu \geq 0} \min _{\substack{\boldsymbol{x} \succeq 0 \\ \boldsymbol{I} \in \mathcal{I}_{\text {CSRA }}}} L(\mu, \boldsymbol{I}, \boldsymbol{x})=L\left(\mu^{*}, \boldsymbol{I}^{*}\left(\mu^{*}\right), \boldsymbol{x}^{*}\left(\mu^{*}, \boldsymbol{I}^{*}\left(\mu^{*}\right)\right)\right),
$$

where $\boldsymbol{x} \succeq 0$ means that $x_{n, k, m} \geq 0 \forall n, k, m, \boldsymbol{x}^{*}(\mu, \boldsymbol{I})$ denotes the optimal $\boldsymbol{x}$ for a given $\mu$ and $\boldsymbol{I}, \boldsymbol{I}^{*}(\mu)$ denotes the optimal $\boldsymbol{I}$ for a given $\mu$, and $\mu^{*}$ denotes the optimal $\mu$. Then we have, for any $(n, k, m)$,

$$
x_{n, k, m}^{*}(\mu, \boldsymbol{I})=I_{n, k, m} p_{n, k, m}^{*}(\mu),
$$

where

$$
p_{n, k, m}^{*}(\mu)= \begin{cases}\tilde{p}_{n, k, m}(\mu) & \text { if } 0 \leq \mu \leq a_{m} b_{m} r_{m} \operatorname{E}\left\{\gamma_{n, k}\right. \\ & \left.U_{n, k, m}^{\prime}\left(\left(1-a_{m}\right) r_{m}\right)\right\} \\ 0 & \text { otherwise }\end{cases}
$$

and

$$
\begin{aligned}
\mu= & \mathrm{E}\left\{U_{n, k, m}^{\prime}\left(\left(1-a_{m} e^{-b_{m} \tilde{p}_{n, k, m}(\mu) \gamma_{n, k}}\right) r_{m}\right)\right. \\
& \left.a_{m} b_{m} r_{m} \gamma_{n, k} e^{-b_{m} \tilde{p}_{n, k, m}(\mu) \gamma_{n, k}}\right\} .
\end{aligned}
$$

To obtain $\boldsymbol{I}^{*}(\mu)$, first define

$$
\begin{aligned}
& V_{n, k, m}\left(\mu, p_{n, k, m}^{*}(\mu)\right) \triangleq \mu p_{n, k, m}^{*}(\mu) \\
& \quad-\mathrm{E}\left\{U_{n, k, m}\left(\left(1-a_{m} e^{-b_{m} p_{n, k, m}^{*}(\mu) \gamma_{n, k}}\right) r_{m}\right)\right\}
\end{aligned}
$$

and

$$
\begin{aligned}
S_{n}(\mu) \triangleq & \left\{(k, m):(k, m)=\underset{\left(k^{\prime}, m^{\prime}\right)}{\operatorname{argmin}} V_{n, k^{\prime}, m^{\prime}}\left(\mu, p_{n, k^{\prime}, m^{\prime}}^{*}(\mu)\right),\right. \\
& \text { and } \left.V_{n, k, m}\left(\mu, p_{n, k, m}^{*}(\mu)\right) \leq 0\right\} .
\end{aligned}
$$

Assuming $S_{n}(\mu)=\left\{\left(k_{i}(n), m_{i}(n)\right)\right\}_{i=1}^{\left|S_{n}(\mu)\right|}$, we have

$$
I_{n, k, m}^{*}(\mu)= \begin{cases}I_{n, k_{i}(n), m_{i}(n)} & \text { if }(k, m)=\left(k_{i}(n), m_{i}(n)\right) \text { for } \\ 0 & \text { some } i \in\left\{1, \ldots,\left|S_{n}(\mu)\right|\right\} \\ 0 & \text { otherwise. }\end{cases}
$$


Here, the vector $\left(I_{n, k_{1}(n), m_{1}(n)}, \ldots, I_{n, k_{\left|S_{n}(\mu)\right|}(n), m_{\left|S_{n}(\mu)\right|}(n)}\right)$ is any point in the unit- $\left(\left|S_{n}(\mu)\right|-1\right)$ simplex, i.e., it belongs to the space $[0,1]^{\left|S_{n}(\mu)\right|}$ and satisfies

$$
\sum_{i=1}^{\left|S_{n}(\mu)\right|} I_{n, k_{i}(n), m_{i}(n)}=1 .
$$

Finally, $\mu^{*}$ (i.e., the optimal value of $\mu$ ) is such that $\mu^{*} \geq 0$ and $\sum_{n, k, m} I_{n, k, m}^{*}\left(\mu^{*}\right) p_{n, k, m}^{*}\left(\mu^{*}\right)=P_{\text {con. }}$.

Let us now define the total optimal allocated power for a given value of $\mu$ as follows:

$$
X_{\text {tot }}^{*}(\mu) \triangleq \sum_{n, k, m} x_{n, k, m}^{*}\left(\mu, \boldsymbol{I}^{*}(\mu)\right) .
$$

Then, the following lemma holds. (The proof is provided in [1].)

Lemma 1. The total optimal power allocation, $X_{\text {tot }}^{*}(\mu)$, is a monotonically decreasing function of $\mu$.

Note that $X_{\text {tot }}^{*}(\mu)$ may not be a continuous function of $\mu$. A sample plot of $X_{\text {tot }}^{*}(\mu)$ and the corresponding value of Lagrangian, i.e., $L\left(\mu, \boldsymbol{I}^{*}(\mu), \boldsymbol{x}^{*}\left(\mu, \boldsymbol{I}^{*}(\mu)\right)\right)$, is shown in Figure 1. From this figure, we observe that $X_{\text {tot }}^{*}(\mu)$ varies continuously in the region (of $\mu$ ) where the optimal allocation, $\boldsymbol{I}^{*}(\mu)$, remains constant and takes a jump (negative) when $\boldsymbol{I}^{*}(\mu)$ changes. In particular, if $\left|S_{n}(\tilde{\mu})\right|>1$ for some $n$ and $\mu=\tilde{\mu}$, then multiple optimal allocations are possible that satisfy (13). However, those different optimal allocations lead to different power consumption levels. The power expended on subchannel $n$ can be any value between $\min _{i \in S_{n}(\tilde{\mu})} p_{n, k_{i}(n), m_{i}(n)}^{*}(\mu)$ and $\max _{i \in S_{n}(\tilde{\mu})} p_{n, k_{i}(n), m_{i}(n)}^{*}(\mu)$, causing a (negative) jump of

$$
\left(\sum_{n} \min _{i \in S_{n}(\tilde{\mu})} p_{n, k_{i}(n), m_{i}(n)}^{*}(\tilde{\mu})-\sum_{n} \max _{i \in S_{n}(\tilde{\mu})} p_{n, k_{i}(n), m_{i}(n)}^{*}(\tilde{\mu})\right)
$$

in $X_{\text {tot }}^{*}(\mu)$ (at $\left.\mu=\tilde{\mu}\right)$. In such cases, we allocate resources according to $\boldsymbol{I}_{\text {CSRA }}^{*} \triangleq \lambda \boldsymbol{I}^{\min }\left(\mu^{*}\right)+(1-\lambda) \boldsymbol{I}^{\max }\left(\mu^{*}\right)$, where

$$
\begin{aligned}
& I_{n, k, m}^{\min }\left(\mu^{*}\right)= \begin{cases}1 & p_{n, k, m}^{*}(\mu)=\min _{i} p_{n, k_{i}(n), m_{i}(n)}^{*}(\mu), \text { and } \\
0 & \text { otherwise, }\end{cases} \\
& I_{n, k, m}^{\max }\left(\mu^{*}\right)= \begin{cases}1 & p_{n, k, m}^{*}(\mu)=\max _{i} p_{n, k_{i}(n), m_{i}(n)}^{*}(\mu) \\
0 & \text { otherwise, }\end{cases}
\end{aligned}
$$

and $\lambda \in[0,1]$ is chosen so that the sum-power constraint is met with equality.

Based on the above discussion, we propose to use bisectionsearch over $\{\mu \geq 0\}$ to find the $\mu^{*}$ at which $X_{\text {tot }}^{*}\left(\mu^{*}\right)=P_{\text {con }}$, stopping at the user-defined search interval $\kappa$. Furthermore, we notice that $p_{n, k, m}^{*}(\mu)$ is a decreasing continuous function of $\mu$ ((8)-(9)) and $\bigcap_{n, k, m}\left\{p_{n, k, m}^{*}\left(\mu^{*}\right) \notin \mathbb{R} \backslash\left[0, P_{\text {con }}\right]\right\}=\emptyset$. Therefore, to obtain $\mu^{*}$, the algorithm does not need to search the values of $\mu$ for which $\tilde{p}_{n, k, m}(\mu)$ combinations do not lie in $\left[0, P_{\text {con }}\right]$ for any $(n, k, m)$. Thus, $\mu^{*} \in\left[\mu_{\min }, \mu_{\max }\right]$, where

$$
\begin{aligned}
\mu_{\max }= & \max _{n, k, m} a_{m} b_{m} r_{m} U_{n, k, m}^{\prime}\left(\left(1-a_{m}\right) r_{m}\right) \mathrm{E}\left\{\gamma_{n, k}\right\} \text { and } \\
\mu_{\min }= & \min _{n, k, m} \mathrm{E}\left\{U_{n, k, m}^{\prime}\left(\left(1-a_{m} e^{-b_{m} P_{\operatorname{con}} \gamma_{n, k}}\right) r_{m}\right)\right. \\
& \left.a_{m} b_{m} r_{m} \gamma_{n, k} e^{-b_{m} P_{\operatorname{con}} \gamma_{n, k}}\right\}
\end{aligned}
$$

are obtained by taking $\tilde{p}_{n, k, m}(\mu) \rightarrow 0$ and $\tilde{p}_{n, k, m}(\mu) \rightarrow P_{\text {con }}$, respectively, for all $(n, k, m)$ in the right side of (10). Table 1 gives the details of an algorithm that solves the CSRA problem.
We now discuss the DSRA problem and its relationship with the CSRA problem. Because it optimizes over both continuous $(\boldsymbol{p})$ and discrete $(\boldsymbol{I})$ variables, the DSRA problem can be recognized as a mixed-integer optimization problem. Due to the high complexity usually associated with mixed-integer optimization problems, we propose an approximate solution to the DSRA problem based on an algorithm with polynomial complexity in $N, K, M$.

Lemma 2. If the solution of the Lagrangian dual of the CSRA problem (7) for a given $\mu$ is such that $\boldsymbol{I}^{*}(\mu) \in\{0,1\}^{N \times K \times M}$, and the corresponding total power is $X_{\mathrm{tot}}^{*}(\mu)$ as in (15), then the solution to the optimization problem

$$
\begin{aligned}
\left(\mathbb{P}^{*}, \mathbb{I}^{*}\right)= & \underset{\substack{\{\mathbb{P} \succeq 0\} \\
\boldsymbol{I} \in \mathcal{I}_{\text {DSRA }}}}{\operatorname{argmax}} \sum_{n, k, m} \mathbb{I}_{n, k, m} \times \\
& \mathrm{E}\left\{U_{n, k, m}\left(\left(1-a_{m} e^{-b_{m} \mathbb{P}_{n, k}, m \gamma_{n}, k}\right) r_{m}\right)\right\} \\
& \text { s.t. } \sum_{n, k, m} \mathbb{I}_{n, k, m} \mathbb{P}_{n, k, m} \leq X_{\text {tot }}^{*}(\mu)
\end{aligned}
$$

satisfies $\mathbb{I}^{*}=\boldsymbol{I}^{*}(\mu)$ and, for every $(n, k, m)$,

$$
\mathbb{P}_{n, k, m}^{*}= \begin{cases}\frac{x_{n, k, m}^{*}\left(\mu, \boldsymbol{I}^{*}(\mu)\right)}{I_{n, k, m}^{*}(\mu)} & \text { if } I_{n, k, m}^{*}(\mu) \neq 0 \\ 0 & \text { otherwise. }\end{cases}
$$

From the above lemma, we conclude that if a $\mu$ exists such that $\left|S_{n}(\mu)\right| \leq 1 \forall n$ and $X_{\text {tot }}^{*}(\mu)=P_{\text {con }}$, then the DSRA problem is solved optimally by the CSRA solution. Recall that $X_{\text {tot }}^{*}(\mu)$ is piece-wise continuous and a discontinuity (or "gap") occurs at $\mu$ when multiple allocations achieving the same optimal value of Lagrangian exist. If the sum-power constraint, $P_{\text {con }}$, lies in one of such "gaps," the optimal allocation for the CSRA problem is given by a convex combination of two elements from the set $\mathcal{I}_{\text {DSRA }}$, and the CSRA solution is not admissible for DSRA. In such a case, we are motivated to choose the sub-optimal DSRA solution $\hat{\boldsymbol{I}}_{\text {DSRA }} \in$ $\left\{\boldsymbol{I}^{\min }(\mu), \boldsymbol{I}^{\max }(\mu)\right\}$ that yields highest utility. Table 1 provides details of the implementation of the proposed sub-optimal DSRA algorithm. Now, the obvious question is: how often do these discontinuities occur? They turn out to be isolated and thus at most countable:

Lemma 3. For any $\tilde{\mu}>0$, there exists $a \delta>0$ such that for all $\mu \in(\tilde{\mu}-\delta, \tilde{\mu}+\delta) \backslash\{\tilde{\mu}\}$, there exists an optimal allocation, $\boldsymbol{I}^{*}(\mu) \in$

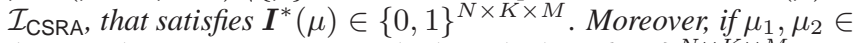
$(\tilde{\mu}-\delta, \tilde{\mu})$, then there exists $\boldsymbol{I}^{*}\left(\mu_{1}\right), \boldsymbol{I}^{*}\left(\mu_{2}\right) \in\{0,1\}^{N \times K \times M}$ such that $\boldsymbol{I}^{*}\left(\mu_{1}\right)=\boldsymbol{I}^{*}\left(\mu_{2}\right)$. The same property holds if both $\mu_{1}, \mu_{2} \in$ $(\tilde{\mu}, \tilde{\mu}+\delta)$.

We now give theoretical bounds on the performances of proposed CSRA and DSRA algorithms.

Lemma 4. Let $\mu^{*} \in[\underline{\mu}, \bar{\mu}]$ be the point where the proposed CSRA algorithm stops, $\hat{U}_{\mathrm{CSRA}}(\underline{\mu}, \bar{\mu})$ be the total utility obtained by the proposed CSRA algorithm, and U USR be the optimal CSRA solution. Likewise, let $\hat{U}_{\mathrm{DSRA}}(\mu, \bar{\mu})$ be the utility achieved by the proposed DSRA algorithm and let $U_{\mathrm{DSRA}}^{*}$ be the utility of the optimal DSRA solution. Then,

and

$$
\left|U_{\mathrm{CSRA}}^{*}-\hat{U}_{\mathrm{CSRA}}(\underline{\mu}, \bar{\mu})\right| \leq(\bar{\mu}-\underline{\mu}) P_{\mathrm{COn}}
$$

$$
\begin{aligned}
& \left|U_{\text {DSRA }}^{*}-\lim _{\underline{\mu} \rightarrow \bar{\mu}} \hat{U}_{\mathrm{DSRA}}(\underline{\mu}, \bar{\mu})\right| \\
& \leq\left(\mu^{*}-\mu_{\min }\right)\left(P_{\mathrm{con}}-X_{\mathrm{tot}}^{*}\left(\boldsymbol{I}^{\min }\left(\mu^{*}\right), \mu^{*}\right)\right) \\
& \leq\left\{\begin{array}{ll}
0 & \text { if }\left|S_{n}\left(\mu^{*}\right)\right| \leq 1 \forall n \\
\left(\mu_{\max }-\mu_{\min }\right) P_{\mathrm{con}} & \text { otherwise }
\end{array} .\right.
\end{aligned}
$$


Compared to the brute-force solution, i.e., by solving the power allocation sub-problem for every possible choice of $\boldsymbol{I} \in \mathcal{I}_{\text {DSRA }}$ (with the same choice of $\kappa$ ) and then selecting the best possible $\boldsymbol{I}$, the proposed DSRA algorithm reduces complexity by the factor $\frac{(K M+1)^{N-1} K M}{(K M+2)}$.

\section{NUMERICAL EVALUATION}

In this section, we numerically investigate the performance of the proposed CSRA and DSRA algorithms in a particular application. We choose the utility function $U_{n, k, m}(x)=x$ for all $(n, k, m)$, so that the objective is to maximize sum-goodput of the system. Furthermore, we consider an uncoded $2^{m+1}$-QAM signaling scheme with MCS index $m \in\{1, \ldots, 15\}, r_{m}=m+1$ bits per symbol, and one symbol per codeword. For MCS index $m$, we select $a_{m}=1$ and $b_{m}=1.5 /\left((m+1)^{2}-1\right)$ because the QAM symbol error rate is proportional to $\exp \left(-1.5 p \gamma /\left((m+1)^{2}-1\right)\right)$ in the high- $(p \gamma)$ regime and $\approx 1$ when $p \gamma=0$. We use the standard OFDM model [14] to describe the (instantaneous) frequency-domain observation made by the $k^{\text {th }}$ mobile user on the $n^{\text {th }}$ subchannel:

$$
y_{n, k}=h_{n, k} x_{n}+\nu_{n, k},
$$

where $x_{n}$ denotes the QAM symbol transmitted by the BS on the $n^{t h}$ subchannel, $h_{n, k}$ is the gain of the $k^{t h}$ user on $n^{t h}$ subchannel, and $\nu_{n, k} \sim$ i.i.d $\mathcal{C N}(0,1)$. Therefore, the exogenous subchannelSNR is given by $\gamma_{n, k}=\left|h_{n, k}\right|^{2}$. The $k^{t h}$ user's channel gains $\boldsymbol{h}_{k}=\left(h_{1, k}, \ldots, h_{N, k}\right)^{T} \in \mathbb{C}^{N}$ (in frequency-domain) are related to the channel impulse response $\boldsymbol{g}_{k}=\left(g_{1, k}, \ldots, g_{L, k}\right)^{T} \in \mathbb{C}^{L}$ via $\boldsymbol{h}_{k}=\boldsymbol{F} \boldsymbol{g}_{k}$, where $\boldsymbol{F} \in \mathbb{C}^{N \times L}$ contains the first $L(<N)$ columns of the $N$-DFT matrix, and where $g_{l, k} \sim \mathcal{C N}\left(0, \sigma_{g}^{2}\right)$ are i.i.d over $(l, k)$. Here, $\sigma_{g}^{2}$ is chosen such that $\mathrm{E}\left\{\gamma_{n, k}\right\}=1$. In the sequel, we use SNR $\triangleq \frac{P_{\text {con }}}{N} \mathrm{E}\left\{\gamma_{n, k}\right\}$ to denote the average available SNR per subchannel.

To model imperfect CSI, we assume that, prior to data transmission, one pilot OFDM symbol is transmitted to every user on every subchannel, from which the corresponding subchannel estimate is computed. In particular, for the $k^{t h}$ user, the pilot observation vector is $\tilde{\boldsymbol{y}}_{k}=\sqrt{p_{\text {pilot }}} \boldsymbol{h}_{k}+\tilde{\boldsymbol{\nu}}_{k} \in \mathbb{C}^{N}$, where the average SNR per subchannel during pilot transmission is $\mathrm{SNR}_{\text {pilot }}=p_{\text {pilot }} \mathrm{E}\left\{\gamma_{n, k}\right\}$. Conditioned on the pilot observation vector, $\boldsymbol{h}_{k}$ is jointly Gaussian with mean $\mathrm{E}\left\{\boldsymbol{h}_{k} \mid \tilde{\boldsymbol{y}}_{k}\right\}$, and covariance matrix $\operatorname{Cov}\left(\boldsymbol{h}_{k} \mid \tilde{\boldsymbol{y}}_{k}\right)$. The resulting $\gamma_{n, k}$ is non-central chi-squared distributed with two degrees of freedom.

We will refer to the proposed CSRA and DSRA algorithms implemented under imperfect CSI as "CSRA-ICSI" and "DSRA-ICSI," respectively. Their utilities will be compared to that of "CSRAPCSI," i.e., CSRA implemented under perfect CSI, which serves as an upper bound, and fixed-power random-user scheduling (FPRUS), which serves as a performance lower bound. FP-RUS schedules, on each subchannel, one user selected uniformly from $\{1, \ldots, K\}$, to which it allocates power $P_{\text {con }} / N$ and the fixed MCS $m$ that maximizes expected sum-goodput. In the plots, the number of OFDM subchannels was $N=64$, the number of users was $K=16$, the impulse response length was $L=2$, and $\kappa=0.3 / P_{\text {con }}$ (recall Table 1). In all plots, goodput values were empirically averaged over 1000 realizations.

Figure 2 plots the subchannel-averaged goodput achieved by the above-described scheduling and resource-allocation schemes for different grades of CSI. The average available subchannel-SNR was kept at $\mathrm{SNR}=10 \mathrm{~dB}$. In this curve, we see that as $\mathrm{SNR}_{\text {pilot }}$ is increased, the performance of CSRA-ICSI and DSRA-ICSI schemes increase from that of FP-RUS to that achieved by the CSRA-PCSI scheme. This is expected because, with increasing $S_{N R} R_{\text {pilot }}$, the BS uses more accurate channel-state information for scheduling and resource allocation, and thus achieves higher goodput. The plot also shows that, even though the proposed CSRA algorithm solves the CSRA problem optimally, and the proposed DSRA algorithm solves the DSRA problem only approximately, their performances almost coincide.

In Figure 3, the top plot shows the subchannel-averaged goodput and the bottom plot shows the subchannel and realization-averaged value of the bound (in (20)) on the optimality gap of the proposed DSRA solution as a function of SNR. The pilot SNR was kept at $\mathrm{SNR}_{\text {pilot }}=-10 \mathrm{~dB}$. In the top plot, we see that, as SNR increases, the difference between CSRA-PCSI and CSRA-ICSI (or, DSRAICSI) increases. However, this difference grows slower than the difference between the CSRA-PCSI and FP-RUS schemes. Interestingly, even for high values of SNR, the performances of CSRA-ICSI and DSRA-ICSI remain almost identical. The bottom plot, which illustrates the average value of $\left(\mu^{*}-\mu_{\min }\right)\left(P_{\text {con }}-X_{\text {tot }}^{*}\left(I^{\min }, \mu^{*}\right)\right)$ over all realizations and subchannels, shows that the loss in subchannelaveraged goodput due to the sub-optimality of the proposed DSRA solution under imperfect CSI is bounded by $7 \times 10^{-3}$ bits/channeluse even when the subchannel-averaged goodput of DSRA-ICSI is of the order of tens of bits/channel-use. This suggests that the bound we provide in Lemma 4 is quite tight at high values of SNR.

\section{CONCLUSION}

In this paper, we considered the problem of joint scheduling and resource allocation (SRA) in downlink OFDMA systems under imperfect channel-state information and an instantaneous sum-power constraint. We considered two scenarios: 1) when subchannel sharing is allowed, and 2) when it is not. For the first scenario, the solution was found using a dual optimization approach. The second scenario resulted in a mixed-integer programming problem for which an approximate solution was found using the solution obtained the first scenario. Practical implementations of the proposed allocation strategies (for both scenarios) were given and their performances were quantified. Numerical results were then presented under a variety of settings. It was found that the proposed imperfect-CSI-based algorithms offer a significant advantage over schemes that do not use any CSI. Moreover, the performance of proposed algorithms in the two scenarios were almost equal, which leads us to conclude that, in OFDMA-based downlink communication systems under imperfect CSI, it is unlikely that the performance gains that result from timesharing of multiple user-MCS combinations within a single subchannel would justify the additional system-level complexity that would be required to implement such time-sharing.

\section{REFERENCES}

[1] R. Aggarwal, M. Assaad, C. E. Koksal, and P. Schniter, "Optimal joint scheduling and resource allocation in OFDMA downlink systems with imperfect channel-state information," submitted to IEEE Transactions on Signal Processing, 2010. http://arxiv.org/abs/1011.0027.

[2] C. Y. Wong, R. S. Cheng, K. B. Letaief, and R. D. Murch, "Multiuser OFDM with adaptive subcarrier, bit and power allocation," IEEE J. Select. Areas In Commun., vol. 17, pp. 17471758, Oct. 1999. 


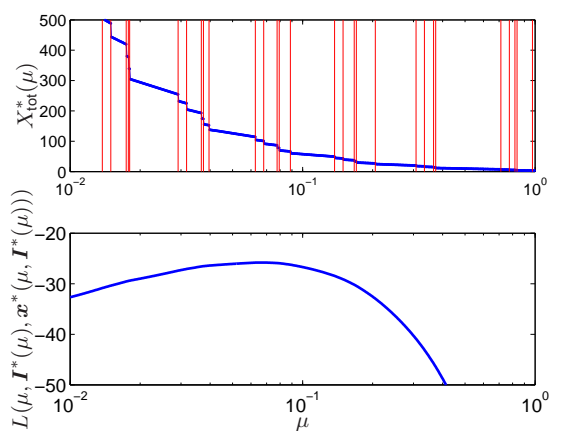

Fig. 1: Average goodput per subchannel versus $\mathrm{SNR}_{\text {pilot. }}$. Here, $N=64, K=16$, and $\mathrm{SNR}=10 \mathrm{~dB}$.

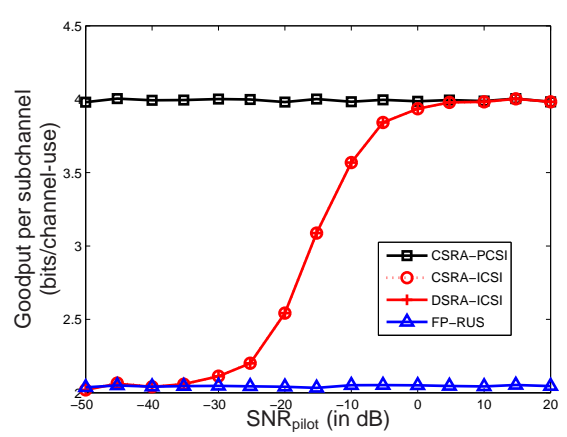

Fig. 2: Average goodput per subchannel versus $\mathrm{SNR}_{\text {pilot. }}$. Here, $N=64, K=16$, and $\mathrm{SNR}=10 \mathrm{~dB}$.
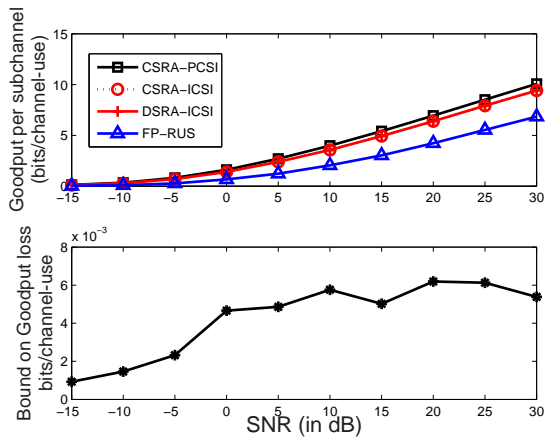

Fig. 3: Subchannel-averaged goodput (top plot) and average bound on optimality gap ((20)) as a function of SNR. Here, $N=64$, $K=16$, and $\mathrm{SNR}_{\text {pilot }}=-10 \mathrm{~dB}$.

[3] J. Jang and K. B. Lee, "Transmit power adaptation for multiuser OFDM systems," IEEE J. Select. Areas In Commun., vol. 21, pp. 171-178, Feb. 2003.

[4] T. J. Willink and P. H. Wittke, "Optimization and performance evaluation of multicarrier transmission," IEEE Trans. Inform. Theory, vol. 43, pp. 426-440, Mar. 1997.

[5] I. Wong and B. Evans, "Optimal downlink OFDMA resource allocation with linear complexity to maximize ergodic rates," IEEE Trans. Wireless Commun., vol. 7, pp. 962 -971, Mar. 2008.

[6] M. R. Souryal and R. L. Pickholtz, "Adaptive modulation with imperfect channel information in OFDM," in Proc. IEEE Int. Conf. Commun., vol. 6, pp. 1861-1865, Jun. 2001.

[7] S. Ye, R. S. Blum, and L. J. Cimini, "Adaptive modulation for variable rate OFDM systems with imperfect channel information," in Proc. IEEE Veh. Tech. Conf., vol. 2, pp. 767-771, 2002.

[8] Y. Yao and G. B. Giannakis, "Rate-maximizing power allocation in OFDM based on partial channel knowledge," IEEE Trans. Wireless Commun., vol. 4, pp. 1073-1083, May. 2005.

[9] I. C. Wong and B. L. Evans, "Optimal resource allocation in the OFDMA downlink with imperfect channel knowledge," IEEE Trans. Commun., vol. 57, pp. 232-241, Jan. 2009.

[10] D. Hui and V. Lau, "Delay-sensitive cross-layer designs for OFDMA systems with outdated CSIT," in Proc. IEEE Wireless Commun. and Networking Conf., pp. 264 -269, Mar. 2007.

[11] A. Ahmad and M. Assaad, "Margin adaptive resource allocation in downlink OFDMA system with outdated channel state information," Proc. IEEE Int. Symposium Personal Indoor Mobile Radio Commun., pp. 1868-1872, 2009.

[12] Y. J. Zhang and S. C. Liew, "Proportional fairness in multichannel multi-rate wireless networks-Part II: The case of timevarying channels with application to OFDM systems," IEEE Trans. Wireless Commun., vol. 7, pp. 3457-3467, Sept. 2008.

[13] S. Boyd and L. Vandenberghe, Convex Optimization. Cambridge University Press, 2004.

[14] J.-J. van de Beek, O. Edfors, M. Sandell, S. Wilson, and P. Borjesson, "On channel estimation in OFDM systems," in Proc. IEEE Veh. Tech. Conf., vol. 2, pp. 815-819, 1995.
Table 1: Algorithmic Implementations

Proposed CSRA algorithm

1. Set $\underline{\mu}=\mu_{\min }, \bar{\mu}=\mu_{\max }$, and $\mu=\frac{\underline{\mu}+\bar{\mu}}{2}$.

2. For each subchannel $n=1, \ldots, N$ :

(a) For each $(k, m)$, use (9)-(10) to calculate $p_{n, k, m}^{*}(\mu)$, and use (11) to calculate $V_{n, k, m}\left(\mu, p_{n, k, m}^{*}(\mu)\right)$.

(b) Calculate $S_{n}(\mu)$ using (12).

3. Use (16) and set $\boldsymbol{I}^{*}(\mu)=\boldsymbol{I}^{\min }(\mu)$

4. Calculate $X_{\text {tot }}^{*}(\mu)$.

5. If $X_{\text {tot }}^{*}(\mu) \geq P_{\text {con }}$, set $\underline{\mu}=\mu$, otherwise set $\bar{\mu}=\mu$.

6. If $\bar{\mu}-\underline{\mu}>\kappa$, go to step 2 ), else proceed.

7. If $X_{\text {tot }}^{*}(\underline{\mu}) \neq X_{\text {tot }}^{*}(\bar{\mu})$, set $\lambda=\frac{X_{\text {tot }}^{*}(\underline{\mu})-P_{\text {con }}}{X_{\text {tot }}^{*}(\underline{\mu})-X_{\text {tot }}^{*}(\bar{\mu})}$, else $\lambda=0$.

8. The proposed allocation is $\hat{\boldsymbol{I}}_{\mathrm{CSRA}}=\lambda \boldsymbol{I}^{*}(\bar{\mu})+(1-\lambda) \boldsymbol{I}^{*}(\mu)$ and the corresponding $\boldsymbol{x}$ is $\hat{\boldsymbol{x}}_{\mathrm{CSRA}}=\lambda \boldsymbol{x}^{*}\left(\bar{\mu}, \boldsymbol{I}^{*}(\bar{\mu})\right)+\left(1^{-}\right.$ $\lambda) \boldsymbol{x}^{*}\left(\underline{\mu}, \boldsymbol{I}^{*}(\underline{\mu})\right)$.

Proposed DSRA algorithm

1. Use the algorithmic implementation of the proposed CSRA solution in to find $\boldsymbol{I}^{*}(\underline{\mu})$ and $\boldsymbol{I}^{*}(\bar{\mu})$.

2. For both $\boldsymbol{I}=\boldsymbol{I}^{*}(\underline{\mu})$ and $\boldsymbol{I}=\boldsymbol{I}^{*}(\bar{\mu})$ (since they may differ)

(a) Set $\mu_{l}=\mu_{\min }, \mu_{u}=\mu_{\max }$, and $\mu=\frac{\mu_{l}+\mu_{u}}{2}$.

(b) For each $n$, calculate $p_{n, k, m}^{*}(\mu)$ where $(k, m)$ is such that $I_{n, k, m}=1$.

(c) Calculate $X_{\text {tot }}^{*}(\boldsymbol{I}, \mu) \triangleq \sum_{n, k, m} I_{n, k, m} p_{n, k, m}^{*}(\mu)$.

(d) If $X_{\text {tot }}^{*}(\boldsymbol{I}, \mu)>P_{\text {con }}$, set $\mu_{l}=\mu$, else set $\mu_{u}=\mu$.

(e) If $\mu_{u}-\mu_{l}>\kappa$, go to step $2 \mathrm{a}$ ), else proceed.

(f) If $X_{\text {tot }}^{*}\left(\boldsymbol{I}, \mu_{l}\right)=X_{\text {tot }}^{*}\left(\boldsymbol{I}, \mu_{u}\right)$, set $\lambda=0$, otherwise set $\lambda=$ $\frac{X_{\text {tot }}^{*}\left(\boldsymbol{I}, \mu_{l}\right)-P_{\mathrm{con}}}{X_{\mathrm{tot}}^{*}\left(\boldsymbol{I}, \mu_{l}\right)-X_{\mathrm{tot}}^{*}\left(\boldsymbol{I}, \mu_{u}\right)}$.

(g) Set $\hat{\boldsymbol{x}}_{\boldsymbol{I}}=\lambda \boldsymbol{x}^{*}\left(\mu_{u}, \boldsymbol{I}\right)+(1-\lambda) \boldsymbol{x}^{*}\left(\mu_{l}, \boldsymbol{I}\right)$, and $\hat{L}_{\boldsymbol{I}}=$ $L\left(\mu, \boldsymbol{I}, \hat{x}_{\boldsymbol{I}}\right)$.

3. The proposed allocation is $\hat{\boldsymbol{I}}_{\mathrm{DSRA}}=\operatorname{argmin}_{\boldsymbol{I} \in\left\{\boldsymbol{I}^{*}(\underline{\mu}), \boldsymbol{I}^{*}(\bar{\mu})\right\}} \hat{L}_{\boldsymbol{I}}$ and the corresponding $\boldsymbol{x}$ is $\hat{\boldsymbol{x}}_{\mathrm{DSRA}}=\hat{\boldsymbol{x}}_{\hat{\boldsymbol{I}}_{\mathrm{DSRA}}}$. 